\title{
Frontières
}

\section{Comment les pères et les mères réinventent-ils leur vie avec un enfant ayant une déficience?}

\section{Diane Pelchat}

Volume 22, numéro 1-2, automne-printemps 2009-2010

Résilience et deuil

URI : https://id.erudit.org/iderudit/045028ar

DOI : https://doi.org/10.7202/045028ar

Aller au sommaire du numéro

\section{Éditeur(s)}

Université du Québec à Montréal

ISSN

1180-3479 (imprimé)

1916-0976 (numérique)

Découvrir la revue

Citer cet article

Pelchat, D. (2009). Comment les pères et les mères réinventent-ils leur vie avec un enfant ayant une déficience ? Frontières, 22(1-2), 58-68.

https://doi.org/10.7202/045028ar
Résumé de l'article

Cette étude qualitative explore les processus d'adaptation/transformation des pères et des mères, sur le plan individuel, conjugal, parental et extrafamilial, ainsi que leurs similitudes et différences dans l'expérience de vivre avec un enfant ayant une déficience motrice cérébrale (DMC). Des entrevues ont été réalisées auprès de 13 pères et de 13 mères d'enfants ayant une DMC. Les résultats révèlent que les pères et les mères perçoivent plus souvent la situation de façon différente que semblable. Cette situation a un potentiel de transformation pour les deux parents. L’importance de la complémentarité entre les pères et les mères est présente dans chacun des sous-systèmes d'adaptation/transformation. Les deux parents cheminent au plan des croyances face à la différence, appliquent leurs nouveaux acquis dans toutes les sphères de leur vie et recherchent une normalisation de leur situation. 


\section{Résumé}

Cette étude qualitative explore les processus d'adaptation/transformation des pères et des mères, sur le plan individuel, conjugal, parental et extrafamilial, ainsi que leurs similitudes et différences dans l'expérience de vivre avec un enfant ayant une déficience motrice cérébrale (DMC). Des entrevues ont été réalisées auprès de 13 pères et de 13 mères d'enfants ayant une DMC. Les résultats révèlent que les pères et les mères perçoivent plus souvent la situation de façon différente que semblable. Cette situation a un potentiel de transformation pour les deux parents. L'importance de la complémentarité entre les pères et les mères est présente dans chacun des sous-systèmes d'adaptation/transformation. Les deux parents cheminent au plan des croyances face à la différence, appliquent leurs nouveaux acquis dans toutes les sphères de leur vie et recherchent une normalisation de leur situation.

Mots clés: similarités et différences pères/ mères - enfant - déficience - processus d'adaptation/transformation - stratégies adaptatives - complémentarité recherche de la normalité.

\section{Abstract}

This qualitative study explored the adaptation/transformation process in mothers and fathers at the individual, marital, parental and extrafamilial levels, and the similarities and differences in their experience of living with a child with cerebral palsy (CP). Interviews were conducted with 13 mothers and 13 fathers of children with CP. The results show that mothers and fathers are more likely to view the situation differently than similarly. For both parents, the situation offers the potential for transformation. Complementarity between mothers and fathers is an important factor in each of the adaptation/transformation subsystems. Both parents embark on a journey changing their beliefs about difference, apply their new knowledge to every aspect of their life, and endeavour to normalize their situation.

Keywords: similarities and differences fathers/mothers - child - disability adaptation/transformation process - adaptive strategies complementarity - quest for normality.

\section{COMMENT LES PÈRES ET LES MÈRES RÉINVENTENT-ILS LEUR VIE AVEC UN ENFANT AYANT UNE DÉFICIENCE?}

Diane Pelchat, Ph. D.,

professeure émérite, Faculté des sciences infirmières, Université de Montréal, Centre de recherche interdisciplinaire en réadaptation du Montréal métropolitain (CRIR) ${ }^{1}$.

\section{PROBLÉMATIQUE ET RECENSION DES ÉCRITS}

La naissance d'un enfant constitue une situation transitionnelle qui provoque à des degrés divers une situation de stress chez les parents. Elle implique de la part des parents la mise en œuvre de stratégies adaptatives pour faire une place à ce nouvel être. Lorsque l'enfant présente un problème de santé, le stress ressenti par les parents est amplifié. Ils doivent alors faire leur deuil de l'enfant «attendu » et s'adapter à l'enfant et à son problème afin de pouvoir vivre affectivement et concrètement avec lui tout en lui fournissant les soins adéquats (Pelchat, 1988). Cela implique qu'ils redéfinissent leurs rôles dans les sphères de la vie familiale et extrafamiliale. Les études relèvent que l'intensité du stress ressenti par les parents d'enfant ayant un problème de santé ne diffère pas des autres familles dans sa nature, mais plutôt dans son intensité. Ainsi, le stress qu'ils vivent est exacerbé par l'imprévisi- bilité des problèmes, les responsabilités et les exigences inhérentes au fait de prendre soin de l'enfant avec un problème (King et al., 1996; Pelchat et al., 2003; Pelchat et al., 1999). Bien que certains parents évoluent relativement bien dans cette situation en développant des stratégies adaptatives pour répondre et s'ajuster aux besoins particuliers de l'enfant, toutes les études affirment que la présence de l'enfant ayant un problème de santé a maintes conséquences dans toutes les sphères de la vie familiale (Pelchat et al., 2003).

Une recension des écrits (Pelchat et al., 2007) met en relief que jusqu'au début des années 1980, peu de recherches ont étudié les différences entre les pères et les mères dans le processus d'adaptation à l'enfant ayant une problématique de santé. La majorité des études sur les familles d'enfant ayant une déficience ont mis l'accent sur les besoins, les perceptions et les comportements des mères. Les études recensées montrent qu'il y a des différences entre les mères et les pères dans leur expérience d'être parents d'un enfant ayant une déficience (Crowe, VanLeit et al., 2000; Pelchat et al., 2003 ; Pelchat et al., 1999). Les mères perçoivent différemment les sources de stress 
(Tamres et al., 2002) et mettent en œuvre un plus large éventail de stratégies pour y faire face. Cependant, l'étude de Pelchat et Bourgeois-Guérin (2009) révèle que les déclencheurs d'incertitude sont les mêmes pour les deux parents, mais leur façon de réagir aux déclencheurs et les choix des

\section{LA MANIÈRE DONT LA PERSONNE}

\section{CHEMINE DANS LE PROCESSUS}

DE DEUIL EST DÉTERMINÉE PAR

\section{SA DYNAMIQUE INDIVIDUELLE}

\section{ET SES RESSOURCES.}

stratégies d'adaptation diffèrent chez les deux parents. Les mères ont toutefois, selon certaines études, un plus haut niveau de stress parental et de dépression que les pères (Bristol et al., 1988; Pelchat et al., 1999). Ces derniers utiliseraient davantage des stratégies cognitives de coping pour s'adapter à la situation (Frey et al., 1989). Les mères utilisent leur réseau de soutien social et les services de santé pour trouver des traitements et contrôler le problème de l'enfant (Frey et al., 1989). Les mères diffèrent des pères en ce qu'elles ont besoin de plus d'informations sur la déficience de l'enfant que les pères et de rencontrer d'autres parents qui vivent la même situation (King et al., 1996; Pelchat et Bourgeois-Guérin, 2009). Les pères auraient plus d'occasions que les mères d'éviter les problèmes avec leur enfant et recourraient davantage au divertissement pour se distancer du problème de l'enfant (Frey et al., 1989).

Depuis la dernière décennie, les résultats des études dépeignent un portrait transformé et moins sombre de la situation des familles d'un enfant atteint d'une déficience, tendant à reconnaître la situation des familles où un enfant présente une déficience, peu importe la structure familiale (Scorgie et Sobsey, 2000). Les chercheurs reconnaissent maintenant la capacité des parents à gérer efficacement et positivement la situation (Pelchat et al., 2002; Crowe et al., 2000; Pelchat et al., 1999). Scorgie et Sobsey (2000) rapportent que certains parents vivent des changements positifs aux plans individuel, interpersonnel et de leurs valeurs spirituelles. Les parents apprennent et se transforment au contact de leur enfant (Lemanek et al., 2000; Pelchat et al., 2004b; Scorgie et Sobsey, 2000). Cependant, peu d'auteurs s'attardent aux apprentissages et aux stratégies d'adaptation utilisées par les mères et les pères d'un enfant ayant un problème de santé. Seuls Pelchat et ses collaborateurs (Pelchat et al., 2004b) ont exploré les nombreux apprentissages des parents d'enfant ayant une trisomie et des infirmières impliquées auprès d'eux.

Même si des études portent sur l'impact de la problématique de santé d'un enfant sur le fonctionnement individuel et familial et sur la manière dont les parents s'y prennent pour faire face à cette situation, à notre connaissance, aucune étude ne traite des différences et des similitudes du processus d'adaptation/transformation des pères et des mères selon leur point de vue. Dans le contexte de la réforme des services de santé qui mise sur la responsabilisation des familles, il est important d'examiner l'expérience des pères et des mères d'enfant ayant une déficience en termes des apprentissages réalisés par chacun afin de mieux cerner comment ils gèrent la situation, s'y adaptent et se transforment.

\section{BUT DE LA RECHERCHE}

L'étude explore le processus d'adaptation/transformation des pères et des mères dans l'expérience de vivre avec un enfant ayant une déficience motrice cérébrale (DMC) dans leurs similitudes et leurs différences aux plans: individuel, conjugal, parental et extrafamilial. Plus spécifiquement, l'étude explore: 1) les différences et les similitudes des perceptions de chacun des parents; 2) les stratégies d'adaptation utilisées par chacun des parents; 3) les transformations vécues par ces derniers.

\section{CADRE DE RÉFÉRENCE : MODËLE CLINIQUE DU PROCESSUS D'ADAPTATION/TRANSFORMATION DU PRIFAM}

Le modèle clinique du Processus d'adaptation/transformation du programme d'intervention interdisciplinaire et familiale (PRIFAM) propose une compréhension novatrice de la dynamique individuelle et familiale à la suite de la naissance d'un enfant avec une déficience ainsi que de l'influence qu'exerce le pro- blème sur la famille et la famille sur le problème. Les mécanismes d'ajustement mis en œuvre par les parents après l'annonce de la déficience de l'enfant s'inscrivent dans un processus qui les mène vers l'adaptation, l'acquisition d'apprentissages et la transformation. Ces constats ont mené au développement du modèle clinique de l'adaptation/transformation (Pelchat, 1989; Pelchat et Lefebvre, 2005).

La prémisse à la base de ce modèle est que l'intensité du stress vécu par les parents est déterminée par un processus d'évaluation de la situation, des stratégies possibles et mises en œuvre ainsi que des ressources disponibles et utilisées par les parents. Ce processus est lui-même influencé par des facteurs internes, externes, contextuels reliés à la déficience de l'enfant ${ }^{2}$. Le modèle du processus d'adaptation/transformation avance que ce n'est pas la gravité du problème qui détermine l'adaptation à la situation, mais bien la perception que les parents ont de celle-ci. Ces perceptions influencent le choix des stratégies des divers sous-systèmes qui permettent ou contraignent l'accomplissement des tâches adaptatives pour progresser vers une adaptation/transformation à la situation. La manière dont la personne chemine dans le processus de deuil est déterminée par sa dynamique individuelle et ses ressources. Ce cheminement vise l'accomplissement de tâches adaptatives, permet de faire le deuil de l'enfant «attendu » et soutient le processus d'adaptation/transformation. La définition du processus d'adaptation/transformation est la suivante: "L'adaptation/transformation est un processus qui permet aux membres de la famille de développer leur capacité de se sentir compétents et confiants dans l'utilisation de leurs ressources et de leur savoir-faire dans la gestion de leur quotidien, de s'autodéterminer et d'actualiser leur nouveau projet de vie» (Pelchat et Lefebvre, 2004a, 2005).

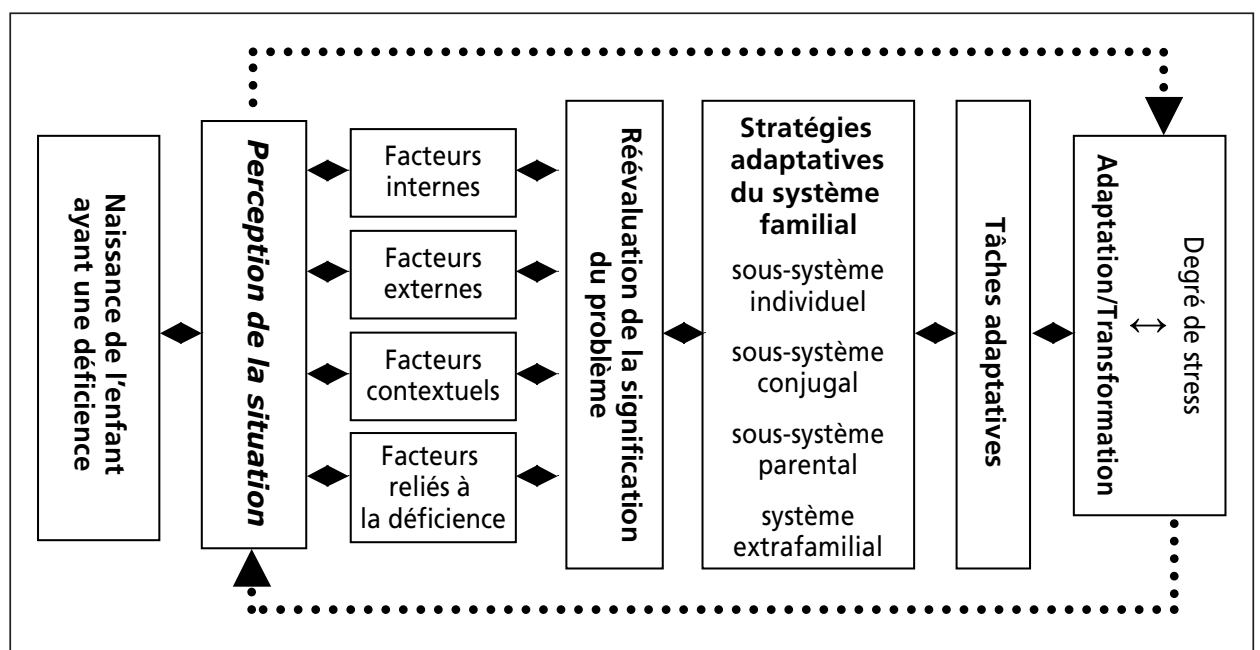




\section{MÉTHODE}

Cette étude qualitative fait appel à une démarche de co-construction de l'expérience et des savoirs de chacun, telle qu'elle est préconisée par Tochon (1996). Les informations ont été recueillies au cours d'entrevues individuelles semi-structurées d'une durée moyenne de 90 minutes, auprès de pères et de mères d'enfants ayant une DMC. Ceux-ci ont été rencontrés simultanément par deux intervieweurs ${ }^{3}$ différents afin d'éviter d'influencer les discours de l'un et de l'autre et de permettre à chacun de s'exprimer librement à propos de leurs expériences et apprentissages respectifs.

\section{ÉCHANTILLON}

Pour obtenir une variété d'information, l'échantillon est constitué selon des critères de diversité de l'expérience, soit l'âge de l'enfant, (entre 14 et 60 mois) et le type d'atteinte (monoplégie, hémiplégie, paraplégie ou quadriplégie), comme le préconisent Glaser et Strauss (1967). L'échantillon est composé de 13 couples de parents qui ont un enfant ayant une DMC recrutés par des personnes ressources dans deux centres hospitaliers, dont l'un est dédié à la pédiatrie, et deux centres de réadaptation des régions du Grand Montréal et des Laurentides. Ils parlent tous le français et habitent dans l'une des deux régions de recrutement. Les pères $(n=13)$ sont âgés entre 29 et 46 ans (moyenne: 38,3 ; écart type: 6,37). La plupart ont un diplôme de niveau secondaire $(41,7 \%)$ ou collégial $(33,3 \%)$ et universitaire $(15,4 \%)$. La majorité des pères travaillent à plein temps $(61,5 \%)$. Les mères sont âgées entre 26 et 43 ans (moyenne: 32,7; écart type: 4,97$)$ et la majorité ont une scolarité de niveau secondaire $(38,5 \%)$, collégiale $(38,5 \%)$ ou universitaire $(23,1 \%)$. Presque la moitié des mères sont à la maison $(46,2 \%)$ et les autres ont des occupations variées, à temps plein $(30,8 \%)$ ou partiel $(23,1 \%)$. Le revenu individuel est de moins de $30000 \$$ pour $46,2 \%$ des pères et pour $69,2 \%$ des mères, et entre $30000 \$$ et $60000 \$$ pour $38,5 \%$ des pères et $30,8 \%$ des mères. Pour les pères, 15,4\% ont un revenu de plus de $60000 \$$. Les couples ont entre un et trois enfants (moyenne: 1,6). Les enfants sont le plus souvent âgés entre 24 et 36 mois $(38,5 \%)$. La majorité des enfants sont atteints de quadraplégie $(61,5 \%)$ et sont nés prématurément $(61,5 \%)$, entre 25 et 37 semaines de grossesse.

\section{ANALYSE DES DONNÉES}

Les verbatims ont été analysés avec le logiciel d'analyse qualitative Nudist et plusieurs rencontres entre les chercheurs de l'étude ont permis d'élaborer les catégories permettant de les classer. La codification et l'analyse des données s'inspirent de la procédure proposée par Merton, Fiske et Kendall (1990). L'analyse a été menée en suivant un mode d'abord inductif puis déductif, se construisant et se raffinant au fur et à mesure du processus d'analyse jusqu'à ce qu'une saturation des catégories soit atteinte. Une analyse de contenu initiale effectuée par unité de sens a permis de déterminer les thèmes émergents du discours et d'en extraire les particularités. Par la suite, les thématiques communes à partir desquelles la catégorisation a été construite furent dégagées. Il en ressort trois principales: la perception, les stratégies adaptatives et les transformations des pères et des mères. La thématique "perception» renvoie à la façon dont les pères et les mères se représentent l'expérience avec l'enfant sur les plans des soussystèmes individuel, conjugal, parental et extrafamilial. Le thème des "stratégies d'adaptation » renvoie aux mécanismes employés par les parents pour s'adapter à la venue et à la présence de l'enfant, sur les plans des mêmes sous-systèmes. Enfin, la catégorie «transformation » renvoie aux apprentissages et aux transformations des pères et des mères sur le plan des croyances, des valeurs et des manières d'être. Cette méthode est complétée par un dernier niveau d'analyse qui a permis de cerner et d'approfondir les convergences et les divergences entre les pères et les mères.

\section{LIMITES DE L'ÉTUDE}

Cette étude fait appel au récit de l'expérience par les acteurs eux-mêmes (parents). Bien que chacun témoigne de plein gré, il est possible que des participants censurent certains commentaires relatifs à ce qu'ils vivent ou ont vécu antérieurement pour différentes raisons. Aussi, il leur est souvent difficile de se remémorer de façon exacte des événements car le temps écoulé et d'autres expériences peuvent avoir modifié leur perception première (Baribeau, 1996). L'utilisation de repères précis durant les entretiens et une attitude d'ouverture à l'autre de la part des intervieweurs permettent d'atténuer l'effet de la perception rétroactive.

\section{RÉSULTATS ET DISCUSSION}

Cette étude qualitative explore les processus d'adaptation/transformation des pères et des mères, sur les plans: individuel, conjugal, parental et extrafamilial, ainsi que leurs similitudes et différences dans l'expérience de vivre avec un enfant ayant une déficience motrice cérébrale (DMC). Les perceptions des pères et des mères les conduisent à utiliser des stratégies adaptatives qui les mènent vers l'adaptation et la transformation. La discussion est élaborée au fil de la présentation des résultats.

\section{PERCEPTIONS ET STRATÉGIES ADAPTATIVES DES PĖRES ET DES MÈRES}

\section{VIE INDIVIDUELLE}

Sur le plan individuel, les similitudes et les différences de points de vue entre les pères et les mères s'expriment dans l'expérience de deuil, la vision des modèles parentaux, leur manière de se percevoir et de percevoir leur conjoint, et dans l'impact du problème de l'enfant sur leur liberté et leurs projets de vie personnels. L'accomplissement de ce processus de deuil a des répercussions dans les autres sous-systèmes et permet aux parents de progresser dans le processus d'adaptation/ transformation.

Bien que les pères et les mères se distinguent dans leur façon de vivre leur deuil de l'enfant «attendu », la grande majorité de ceux-ci vivent une souffrance qui perdure et se manifeste souvent sous la forme d'un deuil récurrent qui refait surface à chaque situation difficile. La mort est souvent utilisée comme épithète pour qualifier l'évanouissement de leurs rêves par rapport à cette naissance: deuil de l'enfant «attendu», deuil d'une paternité ou d'une maternité rêvée: "Pour moi, ça a été comme une mort. Ça a été comme une nouvelle enfant après cette annoncelà. C'est comme si j'avais eu une mort de quelqu'un que j'aimais» (m5). «Il aurait pu nous dire que notre enfant était mort, ça serait la même chose, ça n'aurait pas été plus dur» (p13). Les mères et les pères vivent beaucoup de tristesse: «Une grande tristesse, les femmes, elles pleurent plus que les hommes. Moi, quand j'ai pleuré, j'étais seul» (p10). "C'était horrible. Des moments de folie... J'ai beaucoup pleuré » $(\mathrm{m} 2)$. «Je pense qu'on a été deux semaines à pleurer»(p6). Selon, Singer et ses collègues (1999), l'imprévisibilité de l'événement et l'incapacité de déterminer les causes de la déficience expliquent l'intensité et la durée de leur souffrance.

Le quart des pères et des mères portent un regard positif sur la situation et la considèrent comme une nouvelle expérience à laquelle ils doivent s'adapter, voire comme un défi à relever: "C'est de voir le positif dans le négatif» (m4). "Je vais regarder plutôt les côtés positifs que les côtés négatifs de la vie ou de l'expérience qu'on est en train de vivre avec la petite» (p3). Selon Lazarus et Folkman (1984), lorsque les gens envisagent la situation comme un défi, ils la considèrent alors comme une possibilité de gain, de maîtrise de son environnement et même de croissance personnelle. L'expérience des pères et des mères converge aussi quant au fait de percevoir la déficience de l'enfant comme une mis- 
sion, un devoir à accomplir ou un don de Dieu, surtout lorsque les familles ont de fortes convictions spirituelles comme on le constate dans les extraits suivants: «Ce sont des choses qui sont distribuées par Dieu, il me l'a donné à moi, donc je dois m'occuper d'elle» (m7). «Moi, je me dis... si on l'a eue, parce que le Bon Dieu nous l'a envoyée comme ça, il savait qu'on était des bons parents, puis on va en prendre soin» (p9). Ces parents se définiront comme ayant la capacité de remplir cette mission, considérant que cette épreuve vient mesurer leurs forces, ce qui donne un sens à leur expérience et favorise le processus d'adaptation/transformation (Pelchat et al., 2004b). En ce sens, Boss (2003) relève que cette croyance en un être supérieur favorise la mise en place de stratégies adaptatives visant à donner le maximum à l'enfant et contribue au bienêtre de la famille.

$\mathrm{Au}$ point de vue des stratégies d'adaptation comportementales, les pères et les mères utilisent similairement la stratégie de productivité, l'action: "on fonce, on se prépare, on fait avec, on prend les choses en main» (p3). "On se retrousse les manches, puis on fonce» (m3). Pour d'autres, c'est l'évitement des situations difficiles avec l'enfant, s'évadant pour s'accorder du répit ou pour oublier momentanément le problème. Toutefois, même si la mère dit choisir l'évitement, cela ne semble pas avoir le même poids que pour le père en quantité et en intensité. Pour plusieurs mères, le temps de répit est plus souvent associé à un souhait qu'à un vécu. Dans la mesure où ce comportement ne se cristallise pas, il peut marquer un temps d'arrêt permettant d'accuser le choc de la nouvelle pour ensuite permettre à la personne d'évoluer dans son processus de deuil (Pelchat, 1988).

Les pères et les mères se définissent en se comparant à leur conjoint(e). Lorsque la perception de soi relève des faiblesses, on mentionne souvent les atouts que l'autre possède pour rétablir un équilibre. Cette différence est valorisée comme une complémentarité et davantage exprimée par les mères: "Ce que mon conjoint n'a pas, je l'ai; ou encore ce que je n'ai pas, il le possède» (m1). Elles expriment de façon assez tangible que c'est dans une alliance de leurs atouts individuels qu'ils pourront s'adapter. Les mères vivent davantage leur processus de deuil au niveau émotionnel, qu'elles expriment rapidement et intensément comme le rapporte une mère: «Pleurer me permet de m'adapter» (m1). Celles-ci vivent beaucoup de culpabilité et retournent leur colère contre elles-mêmes. En ce qui concerne la culpabilité, la souffrance des mères et des pères n'est pas si différente, mais elle s'exprime différemment. Pour les mères, elle se vit dans la relation avec l'enfant, ce qui se manifeste parfois dans une surprotection de l'enfant, comme l'exprime une mère: «Le fait d'avoir un enfant handicapé, on a tendance à le surprotéger, puis à se jeter dessus» (m12). Ce qui va dans le sens des propos de Gray (2003) ainsi que de Mendell et ses collègues (2005). Les pères vivent leur souffrance dans leur relation à eux-mêmes, sur un plan plus individuel que relationnel. Leurs réactions sont plus graduelles, plus «contenues » à l'intérieur d'eux-mêmes. La culpabilité semble liée à une blessure d'ordre narcissique, un sentiment d'avoir échoué, tel que le décrivent Aubert-Godard et Scelles (2004). La déficience de l'enfant devient un échec à leur identité masculine et la présence du handicap renvoie au père une image de lui déformée, faite de culpabilité et de honte (Lefebvre, 2006). La honte que vivent les pères renvoie au concept de stigmatisation selon Murphy (1990). Ainsi, un père exprime: "On ne sera jamais une famille standard, on ne sera jamais une famille comme les autres » (p2). Certaines mères sont sensibles au fait que, pour les pères, cette souffrance semble liée à une blessure profonde d'estime de soi, au sentiment d'avoir échoué : «Il s'est senti diminué du fait qu'il s'est dit: "Ah mon Dieu, qu'est-ce qu'on a fait? Pourquoi ça nous arrive à nous autres?" » (m1). Les pères expriment de la colère à l'égard de l'enfant alors que les mères l'expriment indirectement, ce qui est soutenu par les propos de Pelchat (1989).

Les stratégies affectives utilisées par les pères relèvent davantage de la répression des affects, alors que celles des mères visent plutôt leur expression. En effet, celles-ci expriment plus concrètement leur peine (par ex.: pleurs), pour s'adapter, ce qui confirme les résultats de maintes études (Pelchat et al., 2003 ; Gray, 2003). Pour la majorité des pères, la négation ou une attitude d'incrédulité est un mécanisme mis en place à la suite de l'annonce du diagnostic. Les pères semblent avoir plus besoin d'un recul avant d'assimiler l'annonce, comme le relèvent Gray (2003), Heath et Orthner (1999) et Pelchat et al. (2003). Pour leur part, Gage et Kirk (2002) affirment que les hommes semblent prêts moins rapidement que les femmes à devenir parents et mettent plus de temps à réaliser concrètement leur condition de parent, ce qui peut expliquer le délai dans l'expression de leur deuil de l'enfant «attendu».

La stratégie cognitive de recherche d'information est essentielle pour la majorité des mères, ce qui corrobore les études antérieures (King et al., 1996; Gray, 2003). Le type d'information recherchée diffère chez les pères et les mères. Ces dernières cherchent à nommer la déficience ou à la faire valider. Les pères sont plutôt portés vers l'action et la prise de contrôle, ils cherchent à savoir quoi faire avec l'enfant, avec la situation et comment le faire. Seulement le tiers des pères disent rechercher de l'information pour s'adapter à la déficience de l'enfant.

$\mathrm{Au}$ plan des stratégies comportementales, plusieurs mères notent que les pères ont plus recours à l'évitement et à la fuite dans le travail et les loisirs, tendance non relevée par les pères chez leur conjointe. Les mères diront: "Lui, ça peut être différent. Lui, il va aller jouer au hockey, il va aller jouer au baseball, il va travailler, faire du temps supplémentaire, des choses comme ça» (m1). De façon générale, les hommes adopteraient plus souvent une attitude d'évitement face aux difficultés interpersonnelles et aux problèmes de santé, ce qui serait notamment renforcé par les rôles traditionnels de genre (Tamres et al., 2002; Keller et Honig, 2004). Cependant, l'étude de Pelchat (1988) révèle que lorsque la mère prend de la distance face à l'enfant, le père prend la relève en demandant du soutien auprès des membres de sa famille élargie. Il y a ici une complémentarité entre les pères et les mères, comme celle remarquée dans les autres sous-systèmes.

En ce qui concerne la perception de la culture familiale, les pères sont plus souvent en rupture avec le modèle éducationnel paternel et soulignent les qualités $\mathrm{du}$ modèle maternel éducationnel proposé par leur famille d'origine. Un père s'exprime ainsi : «Mon père, il était plutôt négatif puis ma mère est plutôt positive. » (p4), alors que les mères sont davantage en harmonie avec leurs modèles parentaux : «Parce que toujours... dès ma petite enfance, j'ai appris à ne pas seulement aimer les bonnes choses. Je dois toujours accepter les mauvaises choses » (m7). Chez les deux parents, le besoin de rupture avec le modèle éducationnel transmis par la famille d'origine semble être motivé par un modèle relationnel inégal où le père entretient une relation avec son enfant selon un principe d'autorité. Les pères qui rejettent le modèle éducationnel proposé par la famille d'origine semblent ne pas vouloir reproduire un modèle du père autoritaire et ne réinvestiront pas leur relation avec leur père (Pelchat et al., 2003). Inversement, la mère va souvent se rapprocher de ses figures maternelles lorsqu'elle devient elle-même mère et va réinvestir sa relation avec sa mère.

L'expérience des pères et des mères diffère quant à la diminution des loisirs. Les mères évoquent le manque de temps, l'accroissement de leurs responsabilités, 
le manque de ressources financières et le caractère restreint de leur réseau social. Elles font aussi état de l'impact de la déficience de l'enfant sur leurs projets professionnels, qui sont parfois abandonnés, ce qui s'accorde avec les propos d'Ebersold (2007). Les pères ne parlent pas de modification de leurs projets de vie professionnelle et les types d'activités de détente qu'ils pratiquent diffèrent de ceux des mères.

\section{VIE CONJUGALE}

En ce qui a trait à la vie conjugale, l'ensemble des pères et des mères sont conscients du partage inégal des tâches domestiques quotidiennes et des soins reliés à l'enfant: les mères en assument la plus grande partie et certains pères disent être présents et prêts à collaborer sur demande. Voici les propos d'un père: "Je les fais quand elle me dit de les faire $[\ldots] »(\mathrm{p} 2)$. Quoique quelques mères se disent satisfaites du partage des tâches, la majorité des pères et des mères reconnaissent que la prise en charge des tâches parentales est moindre chez le père que chez la mère. Une mère affirme : "Lorsque je parle avec d'autres couples, c'est pas mal la même chose; les hommes en font, mais on aimerait toutes qu'ils en fassent plus, c'est un peu comme ça moi aussi. Il en fait beaucoup, mais on dirait que ce n'est jamais assez. Mais lui trouve qu'il en fait beaucoup» (m4). Ceci confirme l'étude de Pelchat et ses collègues (2003), où les mères disent reconnaître «l'aide» de leur conjoint et apprécier l'effort investi pour les «seconder ». Les pères et les mères relèvent qu'un facteur qui contribue à l'inégalité du partage des tâches est le retour aux rôles traditionnels lorsque l'enfant présente un problème de santé, ce qui confirme les constats de Gray (2003) et de Pelchat et ses collègues (2003). Par contre, les mères expliquent cette inégalité par des éléments contextuels, personnels, relationnels et sociaux comme: l'organisation familiale où le père travaille et la mère demeure à la maison; le manque d'initiative ou d'intérêt du père; le manque de confiance à l'égard des habiletés du conjoint, la prise d'une trop grande place ou de contrôle dans l'exercice de certaines tâches à cause d'un savoir expérientiel que la mère possède. Au-delà de la gestion du quotidien, Neyrand (2000) affirme que souvent les hommes ne s'autorisent pas à occuper une place de père «paternant», qu'ils ressentent à la fois comme étant illégitime et inaccessible par manque d'identification à un père proche de ses jeunes enfants. On pourrait également expliquer l'attitude des mères par le fait que, d'une part, elles vivent plus de culpabilité qu'elles cherchent à soulager en s'occupant de l'enfant et que, d'autre part, la déficience de l'enfant complique le processus de séparation (Pelchat, 1988).

En ce qui concerne la perception de la relation conjugale, presque la moitié des pères et des mères considèrent que cet événement a contribué à la consolidation de leur union ainsi qu'à la capacité d'avoir réussi à se créer ou encore à conserver un espace intime et protégé. Voici leurs propos: "Je pense que ça a créé une corde plus serrée» (m5). "On était bien rapprochés, mais je pense que ça a rapproché notre couple» (p9). Pour ces pères et ces mères, le désir d'avoir un enfant, la qualité de la relation conjugale antérieure à la naissance de l'enfant, l'amour mutuel, la complicité, la complémentarité et le fait de s'orienter vers un objectif commun est garante d'une bonne continuité de vie conjugale. D'autres parents considèrent que cet événement est source de tensions conjugales qui se manifestent dans : les différentes manières des conjoints de réagir à la déficience de l'enfant (mères), l'exigence des soins à l'enfant qui demande beaucoup d'énergie et amène les parents à mettre de côté leur vie conjugale (mères). Les soins et le temps que l'enfant ayant une DMC exige réduit leur espace d'intimité.

L'expérience de plusieurs pères et mères se rejoint au niveau des stratégies d'adaptation conjugales qui s'inscrivent dans une démarche de partenariat où les parents collaborent en complémentarité, dans la reconnaissance et le respect des compétences réciproques, dans l'encouragement respectif et le partage d'un objectif commun, à savoir l'autonomie de l'enfant. Voici leurs propos: "Nous sommes complémentaires. Quand moi, je suis pessimiste, lui, il est optimiste» (m7). «Dans le fond, c'est d'être complémentaires, au lieu d'être toujours pareils $[. .] ».(\mathrm{~m} 1)$. «Il faut se tenir les coudes nous deux ensemble. Quand il y a un problème, il faut le régler, puis il faut que ça marche... faut assumer, puis il faut passer au travers» (p5). Or, comme mentionné antérieurement, la complémentarité joue un rôle important dans le couple (Pelchat et al., 2003).

Seules les mères nomment des sources de tension entre conjoints. Celles-ci incluent les attitudes pessimistes, les propos culpabilisants, les comportements de fuite du conjoint, la peur des réactions de l'autre et l'enfermement sur soi. Selon les mères, le manque de communication et d'ouverture du père face à la situation crée de l'isolement et suscite des interrogations et de l'inconfort. Pelchat (1994) observe que le fait de blâmer l'autre parent, de réprimer l'expression de sa souffrance et de ses inquiétudes par crainte d'augmenter celles de l'autre, entrave la communication et le soutien mutuel.

Les mères se sentent prisonnières des tâches quotidiennes et disent n'avoir plus de temps disponible pour le conjoint. Les pères mentionnent plutôt le manque de loisirs en couple et la surprotection de l'enfant par la mère. Les exigences liées à la condition de l'enfant ayant une déficience contribuent parfois à mettre en péril la relation de couple (Gray, 2003). En ce sens, Simmerman, Blacher et Baker (2001) soutiennent que les couples où les pères assument une large part des soins à l'enfant sont plus satisfaits de l'organisation du quotidien et ont une vie conjugale plus satisfaisante. Ces résultats corroborent ceux d'études précédentes (Pelchat, 1988; Pelchat et al., 2003).

$\mathrm{Au}$ niveau des stratégies d'adaptation, les stratégies communicationnelles sont plus utilisées par les mères que par leur conjoint. Les mères trouvent importante l'attitude compréhensive à l'égard de ce que l'un et l'autre vivent. Cette stratégie semble augmenter en importance avec le vieillissement de l'enfant et le temps qui passe. Les mères portent aussi davantage d'intérêt au bon maintien de leur relation conjugale en ayant recours à un soutien professionnel si nécessaire. Elles valorisent également fortement la complémentarité dans le couple, particulièrement sur le plan du soutien mutuel entre conjoints. Ce qui confirme les constats de Bristol et ses collègues (1988) ainsi que de Pelchat et ses collègues (2003). Les pères indiquent que le dialogue avec leur conjointe pour arriver à des ententes est une stratégie importante pour faire face au stress conjugal. Toutefois, les mères considèrent que le dialogue est une stratégie peu ou pas employée par leur conjoint, comme l'ont relevé Heath et Orthner (1999) ainsi que Pelchat et ses collègues (2003). La qualité $\mathrm{du}$ fonctionnement conjugal et le stress ressenti par les pères sont étroitement liés à la façon dont ils perçoivent le soutien reçu par leur conjointe (Bristol et al., 1988). Cependant, plusieurs écrits affirment que la perception du soutien conjugal est un facteur important du bien-être de la conjointe (Heath et Orthner, 1999). Kersh et ses collègues (2006) confirment que la qualité de la relation conjugale prédit un stress parental moindre et moins de symptômes dépressifs chez les parents.

\section{VIE PARENTALE}

Avec davantage de différences que de similarités, les propos des pères et des mères s'expriment par leur attachement à l'enfant, les soins donnés à celui-ci, le 
maintien de la vie familiale et la modification des rêves et des projets de vie parentaux et familiaux.

De façon similaire, les pères et les mères portent un amour inconditionnel à leur enfant. Ils mentionnent la joie qu'il leur apporte et les atouts que possède cet enfant. Une majorité de mères et la moitié des pères insistent sur la joie que l'enfant leur apporte: "C'est un rayon de soleil» (m9). «C'est ma joie de vivre» (p1). "C'est le plus beau cadeau» (m12). La grande majorité abordent la question des difficultés et des incapacités de l'enfant en le comparant avec un enfant sans problème, faisant un lien avec certaines normes sociales.

La grande majorité des pères et des mères témoignent avec insistance de leur inquiétude face au bien-être, au développement, à l'état de santé de l'enfant et même à sa capacité d'être heureux. Cette préoccupation s'étend sur un continuum allant de l'inquiétude à la surprotection. La plupart des pères et des mères relèvent l'exigence que requièrent les soins et l'attention continue envers l'enfant avec une déficience, l'accroissement de responsabilités et des dérangements liés au comportement de l'enfant. Il importe de souligner que les soins requis par les enfants ayant une DMC sont particulièrement exigeants, les suivis médicaux fréquents et les incertitudes reliées au processus développemental nombreuses.

Sur le plan des stratégies similaires reliées aux soins à l'enfant, on observe des stratégies de réorganisation et d'ajustement de la routine quotidienne pour répondre au rythme de développement de l'enfant, à ses besoins particuliers ou encore pour harmoniser la vie familiale. Une majorité de pères et de mères s'adaptent en renonçant à des rêves tels que des moments de partage de plaisirs, comme faire du sport avec leur enfant. Ces résultats trouvent appui dans l'étude de Graungaard et Skov (2006).

Dans une recherche de la normalité, les parents comparent leur vie à celle de parents d'enfants sans déficience en soulignant qu'eux aussi font face à des difficultés quotidiennes : "Ce que je peux voir avec un enfant normal, avec des parents qui se promènent puis ça va toujours bien $[\ldots]$, tandis que la mienne [...] quand on fait une commande d'épicerie, on pourrait dire une fois sur trois, elle est gentille. À part ça, elle chigne, ça dure dix minutes la crise» (p12). «Il y a des journées que ça va moins bien, il y en a d'autres, c'est comme les personnes qui ont des enfants normaux, aussi, ça ne va pas tout le temps bien non plus. Eux autres aussi sont malades, puis ils sont plus maussades certaines journées » (p3). Ainsi, les parents s'inscrivent dans un espace normatif d'exercice de leur parentalité qui marque peut-être symboliquement la sortie d'un confinement dans une sphère de marginalité (Landsman, 1998). Une autre stratégie d'adaptation importante est celle de leur souci de reconnaissance de leur compétence parentale par eux-mêmes d'abord et par les autres ensuite et parfois par l'enfant lui-même.

$\mathrm{Au}$ plan des nouveaux projets de vie parentale, l'autonomie de l'enfant est le projet qui relie le plus de pères et de mères, mais les mères sont plus préoccupées par cet espoir. En effet, la majorité des mères et la moitié des pères mentionnent qu'en ce qui concerne leur projet parental, leur plus grand défi est de favoriser le développement de l'autonomie de leur enfant. Voici leurs propos: "Je dirais que mon plus grand défi, ça serait qu'un jour elle puisse gagner sa vie. Si elle pouvait finir par gagner sa vie quand elle sera adulte. Je pense que là, j'aurai fait ma job» (p5). "J'aimerais ça, toutes les mères aimeraient ça, que leur enfant marche, même s'il marche juste avec une marchette, j'aimerais ça qu'il soit autonome, qu'il soit capable de s'amuser tout seul, qu'il ait une belle vie, pareil» (m13). Cependant, des différences importantes sont exprimées par rapport aux objectifs poursuivis pour atteindre cette autonomie.

Comme déjà observé sur le plan de la vie individuelle et conjugale, les mères dans leur logique relationnelle sont intéressées à promouvoir, à observer l'aspect relationnel et communicationnel de l'enfant avec les autres et la façon dont les autres le perçoivent et le reçoivent, dans un souci d'acceptation de leur enfant. À ce sujet des mères disent: "Elle aime les gens, donc elle va les approcher, elle va entrer dans la bulle des enfants» (m1). «Moi, c'est surtout le rejet. J'ai peur que ma fille arrive en pleurs de l'école, puis qu'elle me dise: "Oh maman! C'est terrible" " (m12). "C'est sûr que je me fais des idées: "Il n'aura peut-être jamais de petits amis" ou "Qui va venir jouer avec lui ?” " (m6). Pour les pères, toujours dans un souci de recherche de la normalité, ceux-ci stimulent le développement de l'enfant en exigeant de lui des efforts et en lui dressant des limites: "Il faut qu'il se force, il faut qu'il travaille. S'il veut flatter le chat, tu ne lui amènes pas. Il va aller le chercher» (p13). Ils observent et apprécient l'évolution développementale de l'enfant, ce qui semble s'inscrire, encore une fois, dans un souci de normalité: "Quand je la vois, à tous les jours qu'elle progresse, qu'elle fait quelque chose de nouveau, [...] tous les efforts que tu donnes, tu es récompensé par rapport à ça» (p1). Un père rapporte qu'il croit que son fils ne pourra pas être heureux: "Je ne crois pas qu'on puisse être handicapé puis être heureux. Il me semble qu'ils passent à côté de quelque chose» (p13). Indirectement, on peut percevoir une association au bonheur et au bien-être social différente pour les pères et les mères. Les mères dans leur logique relationnelle trouvent gratifiant que leur enfant soit bien reçu ou accepté par les autres et vice-versa, tandis que les pères dans leur logique de recherche de la normalité se sentent valorisés par les progrès au niveau de son développement.

Les pères et les mères se différencient en ce qui concerne la perception de soi comme parent. Les mères utilisent souvent le «je» signifiant leur individualité à l'intérieur de cette fonction parentale. Les pères mentionneront souvent le « on » ou le «nous », signifiant leur fonction parentale. La mère appuiera cette démarcation en disant aussi que les enfants les identifient comme étant des personnes différentes, les jeux seront différents selon le parent, par exemple. Cette difficulté des pères de se différencier des mères serait-elle une manifestation des problèmes qu'ont les pères à trouver leur place et à prendre leur rôle de père? En ce sens, l'étude de Pelchat et ses collègues (2003) révèle que le propos des pères ne permet pas de saisir la représentation qu'ils se font de leur rôle de père. Le père fait alliance avec la mère dans l'exercice de sa fonction parentale vis-à-vis de cet enfant ayant une déficience et la mère souligne qu'elle a sa vision particulière de sa fonction éducatrice et maternelle en tant que parent.

Pour l'organisation de la vie familiale quotidienne, les pères semblent plus affectés par les changements causés par la déficience de l'enfant que les mères qui sont portées à l'ignorer ou à s'oublier, étant surtout interpellées par les besoins de l'enfant. Les pères se sentent harcelés par l'exigence de cette attention soutenue et manifestent un désir de répit. C'est à partir de ces souhaits de plus d'autonomie pour l'enfant que la mère parle indirectement de la lourdeur de sa tâche, du désir d'avoir plus de temps pour s'occuper d'elle. À travers cette revendication du bien-être de l'enfant et parfois de celui de la famille, la mère formule, sans culpabilité, certaines plaintes associées à cette surcharge quotidienne. Les projets du père se situent dans un temps plus éloigné et dans un espace social plus large. Ils se projettent dans un espace différent pour le bien-être, la possibilité de bien remplir leur rôle de pourvoyeur afin d'assurer du confort à leur famille (leur emploi, le 
réaménagement ou l'achat d'une maison...) et la possibilité de bien fonctionner à l'extérieur du cadre familial. Ces résultats vont dans le sens de la vision traditionnelle des rôles parentaux.

Il semble que chez quelques mères les complications au niveau de la santé de l'enfant éveillent une résurgence des émotions associées au processus de deuil de l'enfant «attendu ». Selon certaines mères, ces craintes, associées à la situation particulière de l'enfant ayant une déficience, font en sorte qu'elles surprotègent l'enfant.

Sur le plan des attitudes envers l'enfant, certaines mères évoquent des stratégies qui sont de l'ordre de l'éducation et se manifestent par une attention constante des progrès de leur enfant dans sa vie quotidienne et immédiate. En ce sens, Crowe et Florez (2006) rapportent que les mères organisent et orchestrent les routines journalières en fonction des besoins changeants de l'enfant. Les pères semblent davantage préoccupés par l'établissement de limites à l'enfant, par l'équité pour chacun des enfants du couple ou par la stimulation du développement de l'enfant en exigeant des efforts de lui. Ils vont moins au-devant des désirs de l'enfant que la mère et semblent davantage préoccupés par la vie familiale dans son ensemble. Les pères organisent l'environnement physique de manière à mieux répondre aux besoins de l'enfant qu'ils perçoivent comme normal (Peck et Lillibridge, 2005). Leur implication semble moins relationnelle que celle des mères. Cela témoigne encore une fois de la manière différente qu'ont les pères et les mères de vivre leur parentalité et leur relation avec l'enfant. La mère semble davantage axée sur le micro (le quotidien, la relation) tandis que le père est davantage axé sur le macro (fonctionnement familial, normalité de l'enfant, etc.). Or, ces deux visions apparaissent encore une fois complémentaires. Le père cherche à établir un fonctionnement familial plus normal (Peck et Lillibridge, 2005) et tente de briser la forte symbiose entre la mère et l'enfant en l'incitant à lui accorder moins de temps, à être plus présente auprès des autres enfants et de lui-même (Pelchat et al., 2003).

$\mathrm{Au}$ plan de la différence entre les pères et les mères dans la perception des modifications de leurs projets et rêves parentaux, l'abandon des projets d'activités est perçu comme un deuil par les pères, leur rappelant l'anormalité de leur enfant et les situant dans un espace de marginalité. Cela s'explique notamment par le fait que leurs projets antérieurs à la naissance de l'enfant sont souvent différents de ceux des mères. Par exemple, le rêve de faire des activités sportives avec son fils est plus susceptible d'être abandonné lorsque l'enfant présente une déficience, que celui des mères qui vise le «bonheur» de leur enfant. En outre, comme sont les rêves des pères sont plus souvent liés aux performances de l'enfant et à son inscription dans un environnement social plus large, comme mentionné antérieurement, peut-être sontils plus difficiles à réaliser que ceux des mères. Le désir d'avoir un autre enfant est plus présent chez les pères que chez les mères. Est-ce que les pères restitueraient dans ce désir leur souhait de vivre dans la normalité? Est-ce que les mères, compte tenu de leur relation surinvestie à l'enfant, voient comme impensable l'idée de s'impliquer auprès d'un autre enfant? Ceci s'inscrit dans le même courant d'idées que les études de Bouchard et ses collègues (1994) et Pelchat (1994) qui rapportent les nombreux renoncements auxquels les parents doivent faire face.

\section{VIE EXTRAFAMILIALE}

Les pères et les mères situent leurs propos dans le maintien des liens avec la famille élargie et avec l'entourage, ainsi que dans l'établissement de liens de collaboration avec les professionnels de la santé.

Les pères et les mères expriment de façon similaire que l'annonce du diagnostic enclenche chez les membres de la famille un processus de deuil qui va de la dénégation et la tristesse à l'apprivoisement de la différence de l'enfant. Les membres de la famille élargie et plus particulièrement les grands-parents vivent souvent difficilement l'annonce du diagnostic (Pelchat et al., 2003). Une grand-mère disait: «Lorsque ton enfant a un problème, c'est difficile. Mais lorsqu'il s'agit de l'enfant de ton enfant, c'est doublement difficile.» Le décalage temporel dans la façon dont les membres de la famille élargie vit son processus de deuil de l'enfant «attendu » a pour conséquence, aux dires des parents, qu'ils ne reçoivent pas le soutien qu'ils espéraient obtenir. Pour un peu plus de la moitié des pères et des mères leur expérience converge en ce qui a trait à leur perception du soutien apporté par la famille élargie, de même qu'ils reconnaissent que leur employeur fait généralement preuve de flexibilité et de tolérance à l'égard des absences exigées par les rendez-vous médicaux. Le manque de soutien de la famille élargie et de l'entourage, le manque de spontanéité de leur part au niveau de l'offre de service peuvent être sources de tension. Certains auteurs relèvent que les grandsparents sont la principale source de soutien pour les parents (Blacher et al., 2007) et aussi peuvent devenir une source importante de stress en cas de conflit (Hastings et al., 2002).
Les mères sont plus sensibles que les pères aux réactions et à l'évolution de l'adaptation des membres de la famille élargie à la déficience de l'enfant. Elles observent les signes d'attachement à l'enfant et vivent plus difficilement que les pères les regards stigmatisants des autres, ce qui les porte à s'isoler. Elles s'inquiètent pour l'avenir de l'enfant et craignent l'accentuation des réactions négatives à l'endroit de l'enfant, au fur et à mesure qu'il grandira ayant souvent peur qu'il ne soit pas accepté. De plus, elles semblent plus sensibles que les pères à la persistance d'un malaise de certains membres de la famille élargie. Pourquoi les mères sont-elles plus sensibles au processus de deuil de la famille élargie? Est-ce parce qu'il « résonne» avec leur propre souffrance? Est-ce qu'on peut faire le lien avec les caractéristiques de sensibilité et d'empathie liées au genre féminin?

Sur le plan de l'environnement social, seuls quelques mères et pères disent que leur enfant suscite des regards positifs: "On sort à quelque part, tout le monde dit: "Regarde-la donc si elle est belle!" Le monde nous arrête» (m3). La majorité des parents constatent plutôt que le regard des autres sur leur enfant est surtout empreint de malaise et d'incompréhension et que plusieurs personnes ont tendance à se maintenir à distance d'eux: "Nous sommes allés au Burger King [...] Il y a des gens qui se sont rapprochés pour s'asseoir près de nous. Quand ils ont entendu ma fille s'exprimer, ils ont changé de place» (m1). À ces réactions, quelques pères et mères expliquent que c'est la méconnaissance de leur expérience qui suscite des remarques désobligeantes de la part de l'environnement social. Les réactions du public à l'égard de la déficience de l'enfant sont difficiles à vivre pour la plupart des pères et des mères qui se sentent jugés et blessés par le regard des autres: "C'est sûr que les gens, quand je les vois regarder ma fille, ça me brise le cœur dans le sens que je me dis: "Pourquoi ils se permettent de la regarder de cette façon-là? " Ils ne devraient pas $[. .] ».(\mathrm{~m} 1)$. «Je pense qu'elle a plus peur de leur jugement»(p6). Le regard des autres est souvent stigmatisant, culpabilisant. Certains parents refusent de rester confinés dans cet espace de marginalité en évoquant qu'il s'agit, avant tout, d'un enfant et spécifiant aussi qu'ils sont d'abord des parents et qu'ils méritent, autant eux que l'enfant, d'être considérés comme tels.

Les mères se sentent libérées lorsqu'elles parlent de la déficience de l'enfant qui est vécue comme un secret lourd à porter, un tabou: "C'est comme un peu un secret qu'on a l'impression d'avoir [...] 
J'ai toujours cette impression-là envers quelqu'un quand je ne lui ai pas encore dit, c'est comme si je le cache. Puis quand c'est dit et que c'est bien reçu, je me sens bien là-dedans» (m1, m2). Alors que certains auteurs avancent que le besoin de soutien social est sensiblement aussi important pour les pères que pour les mères (Keller et Honig, 2004), la majorité indiquent que les mères ont davantage recours à l'aide four- des professionnels de la santé ne cadre pas avec leur perception de leur rôle parental et ils peuvent ainsi s'en sentir diminué. Cela pourrait aussi s'expliquer par la socialisation des hommes et des femmes, dont on a parlé antérieurement, la socialisation des femmes étant davantage axée sur les relations interpersonnelles et celles des hommes sur l'autonomie.

Les hommes vivent leurs relations avec

LA MAJORITÉ DES PÈRES ET DES MÈRES S'INSCRIVENT DANS UN PROCESSUS DE CHANGEMENT QUI LES CONDUIT

\section{À TRANSFORMER LEURS CROYANCES, VALEURS ET MANIÈRES D'ÊTRE.}

nie par leur entourage (Frey et al., 1989; Gray, 2003 ; Tamres et al., 2002).

Les pères souhaitent que l'enfant soit perçu comme normal, au-delà de sa déficience par la famille élargie et apprécient le fait de voir l'ouverture à la différence s'installer graduellement chez les membres de leur famille. Certains pères s'inquiètent quant à la possibilité d'avoir du soutien extérieur. Ils font aussi part de leur malaise à parler de la déficience de leur enfant avec leurs collègues. Les pères associent la cause de l'éloignement de l'entourage à la déficience de l'enfant. Un seul s'exprime sur la reconnaissance de l'enfant comme un être digne d'être aimé et accepté et, contrairement aux mères, il affronte le problème. Par contre, selon Pelchat et ses collègues (2003), les pères semblent préférer que l'enfant soit reconnu comme un enfant normal et souhaitent que l'on s'attarde à reconnaître ce qu'il a de semblable avec les autres enfants. Il semble ainsi que ce qui affecte plus particulièrement le père est la mise en évidence de la différence et le constant rappel de ce qui n'est pas normal chez l'enfant, ce qui appuie les propos de Saloviita, Itälinna et Leinonen (2003) affirmant que les pères vivent davantage de stress associé à leurs perceptions de l'acceptation sociale de leur enfant.

Sur le plan extrafamilial, ce n'est pas la stratégie adoptée qui diffère mais la fréquence de son utilisation qui varie. Les mères ont davantage recours à la famille élargie, à l'entourage et aux ressources de la santé et communautaires que les pères. Elles prennent la parole et vont chercher le soutien dont elles ont besoin. Elles font des confidences, parlent de leurs problèmes à des amis ou à des proches. Ces constats sont documentés dans les écrits (Gray, 2003). Nous pouvons émettre l'hypothèse que comme les pères se perçoivent comme une source de soutien pour les mères (Gray, 2003), accepter du soutien les professionnels différemment des mères. Ils disent d'ailleurs de leur conjointe qu'elles sont débrouillardes, persévérantes, patientes et plus ouvertes qu'eux face aux ressources extérieures à la famille. Les pères disent se fier à leur conjointe pour obtenir l'information dont ils ont besoin. Ils se sentent facilement envahis par les intervenants, sont peu disponibles pour prendre en charge les démarches liées aux services et rencontrer les différents professionnels. L'étude de Pelchat (1989) relève que les pères ressentent facilement des émotions négatives par rapport aux professionnels impliqués dans les soins de l'enfant. Aussi, ceux-ci acceptent l'aide afin d'en tirer des bénéfices quantifiables et observables alors que les mères s'y investissent davantage afin d'en tirer un soutien affectif. Cependant, la satisfaction qu'ils affichent de pouvoir parler de leur expérience en groupe, relevée par Pelchat et ses collègues (2003), laisse supposer qu'ils ont aussi besoin de s'ouvrir et d'obtenir davantage de soutien affectif.

\section{TRANSFORMATION DES CROYANCES, DES VALEURS ET DES MANIĖRES D'ÊTRE}

La majorité des pères et des mères s'inscrivent dans un processus de changement qui les conduit à transformer leurs croyances, valeurs et manières d'être. Les transformations qui se réalisent sur le plan individuel, se co-construisent et coévoluent avec des transformations dans les autres sous-systèmes (Pelchat, 1988, 1994; Pelchat et Lefebvre, 2005).

La plupart des parents ont modifié leurs croyances surtout en regard des préjugés liés à la déficience. Ils ont appris à reconnaître les forces, les compétences et le potentiel des personnes ayant des incapacités en observant des personnes adultes atteintes d'une déficience. En démystifiant la différence, ils portent leur attention sur la personne comme n'étant plus limitée à sa seule déficience mais plutôt dans sa normalité. En resituant leur fonction parentale dans la même logique, ils se considèrent d'abord comme les parents d'un enfant dont le lien parental s'ancre dans un espace de normalité. Une transformation de la perception de la déficience de l'enfant s'effectue en valorisant ce que l'enfant peut apporter de joie. Cela permet une évolution dans le processus d'adaptation/ transformation encourageant les parents à valoriser les capacités de développement de leur enfant.

Sur le plan de la relation conjugale, le quart des pères et des mères considèrent que cet événement a contribué à leur rapprochement, à la consolidation de leur union et à la transformation de celle-ci. Ces résultats sont appuyés par Pelchat et ses collègues (1999), Scorgie et Scobsey (2000) et Knafl et Zoeller (2000) qui affirment que, pour certains parents, cette expérience peut parfois se transformer positivement. Une transformation s'opère chez les parents quant aux priorités accordées aux valeurs matérielles et à l'apparence extérieure: ils priorisent la vie familiale et le bien-être des enfants et de la famille qui pourra, quelquefois aller jusqu'au don de soi, à l'abnégation personnelle pour l'éducation des enfants. Certaines attitudes peuvent aussi se transformer lorsqu'un des deux conjoints adopte les valeurs de l'autre après avoir révisé les siennes. Cependant, ce nouveau type de vie et l'engagement des parents envers les enfants, surtout s'ils sont malades, peuvent être qualifiés de lourds.

Concernant la transformation des manières d'être, les pères et les mères accordent moins d'importance au regard des autres et à l'opinion du public et transforment leur attitude en affrontant la situation réelle et en se comparant aux familles d'enfant sans problème de santé. La transformation d'attitude à l'égard de la déficience prend souvent l'allure d'une résistance à la stigmatisation de l'enfant et d'eux-mêmes comme parents. Morse et ses collègues (2000) affirment que le processus de normalisation se réalise lorsque la famille travaille activement à identifier des façons d'expliquer que leur vie n'est pas si différente de celle des familles d'enfant sans problème chronique. Les pères et les mères endossent une lentille de la «normalité» (Robinson, 1993) pour définir leurs relations parentales, conjugales et sociales pour assumer la condition chronique de l'enfant.

En ce qui concerne les différences, les résultats mettent en évidence que c'est parfois la spécificité de la transformation, en termes de croyances, de valeurs et de manières d'être, qui différencie les pères 
des mères. Certains parents l'expliquent par rapport aux différences de personnalité et d'autres à partir des rôles parentaux sexués. Les occasions d'apprentissage sont différentes comme une mère l'explique: «la leçon est différente pour les deux».

L'évolution des croyances, des valeurs et des manières d'être des mères semble davantage se manifester que chez les pères. Elle se manifeste dans la tendance des mères à valoriser le moment présent, à diminuer leurs attentes envers l'enfant, à ralentir le rythme de la vie quotidienne et ainsi à mieux profiter de la vie. Elles acquièrent un plus grand respect et une plus grande valorisation de ce qu'elles sont tout en respectant leurs limites. Elles apprennent à prendre soin d'elles-mêmes et, parfois, à se défaire de leur sentiment de responsabilisation à l'égard des autres. La plupart des mères indiquent que leur expérience leur a permis de développer leur sentiment de confiance en leurs compétences et en leurs ressources. Ces apprentissages réalisés par les mères confirment les résultats de Pelchat et ses collègues (2004b). Du côté des pères, la moitié acquièrent et développent certaines qualités et transforment parfois leur façon d'être. En effet, l'expérience de vie avec l'enfant ayant une déficience amène certains pères à manifester leur sensibilité et à devenir plus conscients de la fragilité de la vie et de l'incertitude qui en découle. D'autres pères mentionnent avoir développé de la persévérance, de la ténacité et le désir de se dépasser au contact de l'enfant: "Je me suis senti grandir au contact de ma fille» (p3). Les propos des pères et des mères tendent aussi à montrer que la venue et la présence de l'enfant ayant une déficience encouragent le développement d'une nouvelle maturité chez les pères. Les mères sont plus nombreuses à transformer leur manière d'être sur le plan personnel. L'étude de Hastings et ses collègues (2005) relève que les mères notent généralement davantage de contributions positives liées à la présence de leur enfant ayant une déficience que les pères, car elles sont plus présentes auprès de l'enfant ayant une déficience que le père qui prend plus de temps pour observer la contribution de celui-ci auprès de la famille.

Sur le plan conjugal, ce sont surtout les mères qui disent avoir appris à accepter et à respecter la différence dans leur couple. Elles soulignent aussi le co-apprentissage avec leur conjoint dans la modification de leurs priorités et de leurs valeurs. Cette situation permet au couple de réviser les valeurs héritées du patrimoine familial et culturel pour adopter d'autres valeurs et croyances qui font l'assentiment des deux conjoints, qui en feront une valeur familiale. C'est ce que Daly (2004) définit comme une culture parentale «culture du moment» qui évolue et se transforme au contact mutuel des parents et des enfants.

La transformation se manifeste notamment au niveau du sentiment de compétence parentale quant aux besoins et aux exigences découlant de la déficience de l'enfant. Les résultats indiquent que les mères sont davantage portées que les pères à revaloriser leurs compétences parentales. Certaines mères relèvent que leur apprentissage auprès de l'enfant permet le transfert de savoirs vers d'autres enfants de la famille. En ce qui a trait à la transformation des attitudes envers l'enfant, la mère va plutôt investir de l'énergie à se questionner sur les besoins, la maladie de l'enfant et ne questionnera pas ou peu son rapport avec l'enfant alors que le père le fera davantage. Il fera la découverte de son rapport intime avec cet enfant dans un souci de transformer ou d'améliorer son comportement. Il y a une prise de conscience chez les pères d'une meilleure maîtrise de ses habiletés dans son comportement envers l'enfant se manifestant notamment par l'acquisition de la patience et par un sentiment d'admiration envers l'enfant. Aussi, les pères généralisent leurs apprentissages en dépassant les frontières familiales et en prenant une position sociale qui s'inscrit dans cette nouvelle interprétation du respect de la déficience qu'ils ont acquise.

Les compétences acquises sur le plan individuel par les mères se manifestent dans diverses situations sociales, comme celles d'avoir amélioré sa sociabilité et d'avoir acquis de la débrouillardise et de l'assurance auprès des professionnels de la santé. De leur côté, les pères extrapolent ces savoirs et transforment plutôt croyances, valeurs et manières d'être face à la déficience en général dans un espace social plus large (participation à des comités de défense des droits, contributions monétaires à des organismes, prise de positions et défense du respect des personnes ayant une déficience).

La différence concernant la transformation est aussi liée à l'âge de l'enfant. Il semble que la chronologie pour la transformation est différente chez les pères et les mères. Cette transformation se réalisera plus rapidement chez les pères que chez les mères. D'un point de vue global, les résultats tendent à montrer que la transformation des valeurs est plus fréquente chez les mères ayant un enfant âgé de plus de trois ans que chez celles ayant un enfant plus jeune. À l'opposé, les pères dont l'enfant est plus jeune évoquent plus fréquemment la transformation de certaines valeurs que ceux ayant un enfant plus vieux.
En résumé, les pères et les mères appliquent leurs nouveaux acquis dans toutes les sphères de leur vie. Sur le plan individuel, les mères développent un plus grand respect et une plus grande valorisation de ce qu'elles sont en passant par le respect de leurs limites alors que les pères disent avoir développé la patience, la persévérance. Sur le plan parental, les mères et les pères développent de nouvelles habiletés parentales, apprennent à faire confiance à leur enfant et respectent les valeurs éducatives des autres parents. Sur le plan conjugal, ils apprennent à respecter leurs différences. En ce qui concerne les professionnels de la santé, ils utilisent les apprentissages et leur transformation afin de répondre à leurs besoins. À travers ce processus de transformation, il y a de la part des pères et des mères une recherche de normalisation de leur situation de vie avec un enfant ayant une déficience.

\section{RECOMMANDATIONS}

Cette étude démontre l'importance de sensibiliser les professionnels de la santé à soutenir les parents dans l'accomplissement d'une tâche adaptative des plus importantes qui est d'assumer leur espace de normalité. Les parents se sentent stigmatisés par leur entourage et ceux-ci doivent, par une recherche de la normalité, inscrire leur rôle de parents dans un espace normatif sur le plan individuel, parental, conjugal et en relation avec la famille élargie, l'entourage, l'environnement social et avec les professionnels de la santé. Par leurs attitudes, les professionnels de la santé peuvent être des agents de stigmatisation ou de normalisation de la situation des parents. Les pères prennent plus de temps à s'attacher à leur enfant et ont des attentes parfois irréalistes, ceux-ci peuvent se désinvestir lorsqu'ils s'aperçoivent que leurs attentes ne se réalisent pas. Aussi, ils adoptent plus facilement une stratégie de fuite alors que la mère investit son énergie dans les soins de son enfant. Il est frappant de constater que les stratégies des mères apparaissent plus adéquates aux yeux des mères, des pères euxmêmes et des professionnels de la santé. Cela pourrait avoir comme effet pervers de décourager les pères, qui se désinvestiraient davantage en réaction à cette impression. Il est possible que plus les pères se sentent jugés, plus ils deviennent méfiants et se retirent. Or, plus ils se retirent, plus les professionnels adoptent des stratégies visant à favoriser l'implication parentale plutôt que d'essayer de comprendre l'expérience des pères. Tout en impliquant les pères dès l'annonce, il est important de respecter leur rythme dans leur évolution du processus d'adaptation/transformation. 
L'étude permet une meilleure compréhension du processus d'adaptation/ transformation des pères et des mères, de la dynamique individuelle et familiale qui s'installe à l'arrivée de l'enfant, des effets de marginalisation potentielle de ces parents et des façons différenciées des pères et des mères de vivre leur deuil et de s'adapter à l'expérience de vivre avec un enfant ayant une déficience. Une différence importante se situe dans la poursuite du projet commun d'autonomie de l'enfant. Pour les mères, celui-ci est relié à l'aspect relationnel et communicationnel de l'enfant avec les autres, c'est-à-dire à son acceptation par les autres. Pour les pères, l'autonomie de l'enfant est reliée à son processus développemental et aux progrès réalisés par celui-ci. Cette divergence fait en sorte que les pères et les mères adoptent des stratégies différentes et complémentaires auprès de l'enfant. Bien que les pères et les mères perçoivent la situation problématique de façon plus souvent différente que semblable dans l'ensemble des sphères et que les mères semblent posséder un plus grand registre de stratégies adaptatives, cette situation a un potentiel de transformation pour les deux parents. La très grande majorité des pères et des mères s'inscrivent dans un processus de changement qui les conduit à transformer leurs croyances, valeurs et manières d'être dans toutes les sphères de leur vie. L'importance de la complémentarité et de la complicité entre les pères et les mères, qui sont présentes dans chacun des sous-systèmes d'adaptation/transformation, de même que la recherche de la normalité par l'adaptation à la différence dans l'ensemble des sous-systèmes constituent un résultat important. Cette étude renforce l'importance d'une intervention familiale systémique qui tient compte de l'interinfluence de chacun de ses membres et leurs besoins respectifs. En somme, elle fournit des indicateurs pouvant être appliqués à la formation des professionnels afin de favoriser le processus d'adaptation/transformation des pères et des mères.

\section{Bibliographie}

AUBERT-GODARD, A. et R. SCELLES (2004). «Écouter les pères, leur ménager une place, une mesure de prévention pour le devenir de l'enfant handicapé et de ses proches », Pratiques psychologiques, vol. 10, $\mathrm{n}^{\circ} 2$, p. $169-185$.

BARIBEAU, C. (1996). "La rétroaction vidéo et la construction des données ", Revue des sciences de l'éducation, vol. 22, $\mathrm{n}^{\circ}$ 3, p. 577-598.

BLACHER, J., K.A. FEINFIELD et B.R. KRAEMER (2007). "Supporting families who have children with disabilities ", dans A. CARR, G. O'REILLY, P.N. WALSH et
J. MCEVOY (dir.), The Handbook of Intellectual Disability and Clinical Psychology Practice, New York, Routledge/Taylor et Francis Group, p. 303-335.

BOSS, P. (2003). Family Stress Management, $2^{\mathrm{e}}$ éd., Thousand Oaks, Sage Publications.

BOUCHARD, J-M, D. PELCHAT, P. BOUDREAULT et M. GRATTON-LALONDE (1994). Déficiences, incapacités et handicaps: processus d'adaptation et qualité de vie de la famille, Montréal, Éditions Guérin Universitaire.

BRISTOL, M.M., J.J. GALAGHER et E. SCHOPLER (1988). "Mothers and fathers of young developmentally disabled and nondisabled boys: Adaptation and spousal support », Developmental Psychology, vol. 24, $\mathrm{n}^{\circ} 3$, p. 441-451.

CROWE, T.K. et S.I. FLOREZ (2006). "Time use of mothers with school-age children: A continuing impact of a child's disability ", The American Journal of Occupational Therapy, vol. $60, \mathrm{n}^{\circ} 2$, p. 194-203.

CROWE, T.K., B. VANLEIT et K.K. BERGHMANS (2000). "Mothers' perceptions of child care assistance: The impact of a child's disability », The American Journal of Occupational Therapy, vol. 54, $\mathrm{n}^{\circ} 1$, p. $52-58$.

DALY, K. (2004). «L'évolution de la culture parentale ", Insitut Vanier de la famille, en ligne, <http://www.vifamily.ca/library/cft/ parenting_fr.html>, consulté le 2010-07-22.

EBERSOLD, S. (2007). Parents, professionnels, face au dévoilement du handicap, Dires et regards, Saint-Agne, Erès.

FREY, K.S., R.R. FEWELL et P.F. VADASY (1989). «Parental adjustment and changes in child outcome among families of young handicapped children", Topics in Early Childhood Special Education, vol. 8, n 4 , p. $138-57$

GAGE, J.D. et R. KIRK (2002). « First-time fathers: Perceptions of preparedness of fatherhood ", Canadian Journal of Nursing, vol. 34, no 4, p. 15-24.

GLASER, B.G. et A.L. STRAUSS (1967). The Discovery of Grounded Theory, Strategies for Qualitative Research, Chicago, Aldine.

GRAUNGAARD, A.H. et L. SKOV (2006). "Why do we need a diagnosis? A qualitative study of parents' experiences, coping and needs, when the newborn child is severely disabled », Child: Care, Health and Development, vol. 33, n 3, p. 296-307.

GRAY, D.E. (2003). "Gender and coping: The parents of children with high functioning autism ", Social Science and Medicine, vol. 56, p. 631-642.

HASTINGS, R.P., A. BECK et C. HILL (2005). "Positive contributions made by children with an intellectual disability in the family: Mothers' and fathers' perceptions ", Journal of Intellectual Disabilities, vol. 9, n 2, p. 155-165.

HASTINGS, R., H. THOMAS et N. DELWICHE (2002). "Grandparent support for families of children with Down's syndrome»,
Journal of Applied Research in Intellectual Disabilities, vol. 15, $\mathrm{n}^{\circ}$ 1, p. 97-104.

HEATH, D.T. et D.K. ORTHNER (1999). "Stress and adaptation among male and female single parents ", Journal of Family Issues, vol. 20, $\mathrm{n}^{\circ} 4$, p. 557-587.

KELLER, D. et A.S. HONIG (2004). «Maternal and paternal stress in families with school-aged children with disabilities", American Journal of Orthopsychiatry, vol. 74, no 3, p. 337-348.

KERSH, J., T.T. HEDVAT, P. HAUSERCRAM et M.E. WARFIELD (2006). "The contribution of marital quality to the well-being of parents of children with developmental disabilities », Journal of Intellectual Disability Research, vol. 50, n 12 , p. 883-893.

KING, G.A., S.M. KING et P.L. ROSENBAUM (1996). «How mothers and fathers view professional caregiving for children with disabilities", Developmental Medicine and Child Neurology, vol. 38, $\mathrm{n}^{\circ} 5$, p. 397-407.

KNAFL, K. et L. ZOELLER (2000). « Childhood chronic illness: A comparison of mothers' and fathers' experiences », Journal of Family Nursing, vol. 6, n 3, p. 287-302.

LANDSMAN, G.H. (1998). "Reconstructing motherhood in the age of "perfect" babies: Mothers of infants and toddlers with disabilities», Signs: Journal of Women in Culture and Society, vol. 24, n ${ }^{\circ}$ 1, p. 69-97.

LAZARUS, R.S. et S. FOLKMAN (1984). Stress, Appraisal and Coping, New York, Springer Publishing Compagny.

LEFEBVRE, G. (2006). Attachement, handicap et paternité, Paris, Fondation pour l'Enfance, lettre $\mathrm{n}^{\circ} 46$.

LEMANEK, K.L., M.L. JONES et B. LIEBERMAN (2000). "Mothers of children with spina bifida: Adaptational and stress processing », Children's Health Care, vol. 29, $\mathrm{n}^{\mathrm{o}} 1$, p. 19-35.

MENDELL, D., R. CURTIS, M. GOLD et S. HARIE (2005). "Anaphylaxis: How do you live with it? ", Health and Social Work, vol. $30, n^{\circ} 4$, p. 325-335.

MERTON, R.K., M. FISKE et P.L. KENDALL (1990). The Focused Interview: A Manual of Problems and Procedures, New York, The Free Press.

MORSE, J.M., S. WILSON et P. JANICE (2000). "Mothers and their disabled children: Refining the concept of normalization ", Health Care for Women International, vol. 21, p. 659-676.

MURPHY, A. (1990). «Human responses to transitions: A holistic nursing perspective", Holistic Nursing Practice, vol. 4, n 3, p. 1-7.

NEYRAND, G. (2000). L'enfant, la mère et la question du père, Un bilan critique de l'évolution des savoirs sur la petite enfance, Paris, Presses universitaires de France.

PECK, B. et J. LILLIBRIDGE (2005). « Normalization behaviours of rural fathers living with chronically-ill children: An Australian experience", Journal of Child Health Care, vol. $9, n^{\circ} 1$, p. 31-45. 
PELCHAT, D. (1994). «L'annonce de la déficience et processus d'adaptation de la famille", Handicap, médecine, éthique, Les cahiers de l'Afrée, $\mathrm{n}^{\circ}$ 6, Montpellier, p. $81-88$.

PELCHAT, D. (1988). Programme d'intervention précoce auprès des parents d'un enfant atteint d'une déficience physique, Montréal, Université de Montréal, en ligne, $<$ http://www.scinf.umontreal.ca/famille/ aaDocuments/Phd88.pdf $>$.

PELCHAT, D., J. BISSON, N. RICARD, M. PERREAULT et J.M. BOUCHARD (1999). «The longitudinal effects of an early family intervention program on the adaptation of families of children with a disability », International Journal of Nursing Studies, vol. 36, $\mathrm{n}^{\circ} 6$, p. 465-477.

PELCHAT, D., H. LEFEBVRE et C. DAMIANI (2002). " Deuil - Appropriation de compétences - Transformation », Pratiques psychologiques, vol. 1, p. 41-52.

PELCHAT, D., H. LEFEBVRE et M. PERREAULT (2003). "Differences and similarities between mothers' and fathers' experiences of parenting a child with a disability", Journal of Child Health Care, vol. $7, \mathrm{n}^{\circ} 4$, p. 231-247.

PELCHAT, D. et H. LEFEBVRE (2004A). "A holistic intervention program for families with a child with a disability », Journal of Advanced Nursing, vol. 48, $\mathrm{n}^{\circ}$ 2, p. 124-131.

PELCHAT, D., H. LEFEBVRE, M. PROULX, J. BOUCHARD, M. PERREAULT et L. BOUCHARD (2004B). "Acquisition de savoirs professionnels et de savoirs parentaux dans le cadre du programme d'intervention PRIFAM », Revue internationale de l'éducation familiale, vol. 8, $\mathrm{n}^{\circ}$ 2, p. 53-72.

PELCHAT, D. et H. LEFEBVRE (2005). Apprendre ensemble, Le PRIFAM, pro- gramme d'intervention interdisciplinaire et familiale, Montréal, Chenelière/McGrawHill.

PELCHAT, D., H. LEFEBVRE et M.J. LEVERT (2007). "Gender differences and similarities in the experience of parenting a child with a health problem: Current state of knowledge ", Journal of Child Health Care, vol. $11, \mathrm{n}^{\circ} 2$, p. 112-131.

PELCHAT, D., et V. BOURGEOIS-GUÉRIN (2009). «Incertitude et stratégies d'adaptation des pères et de mères lors du processus de l'annonce de la Déficience Motrice Cérébrale ", Recherche en soins infirmiers, vol. 96, p. 41-51.

ROBINSON, C.A. (1993). "Managing life with a chronic condition: The story of normalization ", Qualitative Health Research, vol. 3, no 1, p. 6-28.

SALOVIITA, T., M. ITÄLINNA et E. LEINONEN (2003). "Explaining the parental stress of fathers and mothers caring for a child with intellectual disability: A double ABCX model ", Journal of Intellectual Disability Research, vol. 47, nº 4, p. 300-312.

SCORGIE, K. et D. SOBSEY (2000). «Transformational outcomes associated with parenting children who have disabilities », Mental Retardation, vol. 38, $\mathrm{n}^{\circ} 3$, p. 195-206.

SIMMERMAN, S., J. BLACHER et B.L. BAKER (2001). "Fathers' and mothers' perceptions of father involvement in families with young children with a disability », Journal of Intellectual and Developmental Disability, vol. 26, no 4, p. 325-338.

SINGER, L.T., A. SALVATOR, S. GUO, M. COLLIN, L. LILIEN et J. BALEY (1999). «Maternal psychological distress and parenting stress after the birth of a very lowbirth-weight infant», The Journal of the
Amercan Medical Association, vol. 281, no 9, p. 799-805.

TAMRES, L.K., D. JANICKI et V.S. HELGESON (2002). «Sex differences in coping behavior: A meta-analytic review and an examination of relative coping ", Personality and Social Psychology Review, vol. 6, $\mathrm{n}^{\mathrm{0}}$ 1, p. 2-30.

TOCHON, F.V. (1996). «Rappel stimulé, objectivation clinique, réflexion partagée. Fondements méthodologiques et applications pratiques de la rétroaction vidéo en recherche et en formation", Revue des sciences de l'éducation, vol. 22, nº 3, p. 467-502.

\section{Notes}

1. Cette recherche a été subventionnée par le Conseil de recherche en sciences humaines du Canada (CRSH).

2. Les facteurs internes sont reliés à l'individu: la connaissance du problème avant l'arrivée de l'enfant, l'histoire et la personnalité de l'individu, l'âge, le sexe, les problèmes personnels antérieurs et le projet de vie. Les facteurs externes concernent les sous-systèmes familiaux et extrafamiliaux: les dynamiques conjugales, parentales et familiales antérieures à la naissance de l'enfant, les projets de vie conjugaux, parentaux et familiaux, les attitudes et le soutien de la famille élargie. Les facteurs contextuels sont reliés à la famille et aux types de famille, leurs caractéristiques socioéconomiques et leur culture d'origine; à l'intervention et à la relation avec les professionnels de la santé. Enfin, la perception de la situation est également influencée par des facteurs reliés à la déficience elle-même.

3. La chercheure principale a effectué la moitié des rencontres avec les parents. Une agente de recherche détentrice d'une maîtrise et formée par la chercheure principale a effectué l'autre moitié des rencontres avec les parents. 